Supporting information for:

\title{
Probing the Catalytically Active Region in a Nanoporous Gold Gas Diffusion Electrode for Highly Selective Carbon Dioxide Reduction
}

Aidan Fenwick, ${ }^{1,2 *}$ Alex J. Welch, ${ }^{2,3 *}$ Xueqian Li, ${ }^{2,3}$ Ian Sullivan, ${ }^{1,3}$ Joseph S. DuChene, ${ }^{4}$ Chengxiang Xiang, ${ }^{1,3}$ and Harry A. Atwater ${ }^{2,3 \dagger}$

${ }^{1}$ Department of Chemistry, California Institute of Technology, Pasadena, CA 91125, USA

${ }^{2}$ Liquid Sunlight Alliance, California Institute of Technology, Pasadena, CA 91125, USA

${ }^{3}$ Department of Applied Physics and Material Science, California Institute of Technology, Pasadena, CA 91125, USA

${ }^{4}$ Department of Chemistry, University of Massachusetts Amherst, MA 01003, USA

*These authors contributed equally to this work

Corresponding Author:

$\dagger$ E-mail: haa@,caltech.edu

\section{Experimental:}

\section{Materials:}

Potassium carbonate (99.995\% trace metal basis, Sigma Aldrich), Nitric Acid (70\%, purified by redistillation, $>99.999 \%$ trace metals basis, Sigma Aldrich) Si [p-type, 0-10 $\Omega$ cm, (100) orientation, 620 $\pm 25 \mu \mathrm{m}$ thick, University Wafers], Pt gauze (99.9\%, 52 mesh woven from $0.01 \mathrm{~mm}$ wire, Alfa Aesar) and Copper(II) sulfate ( $\geq 99 \%$, Sigma Aldrich) were used without modification unless otherwise noted. The carbon paper substrate (Sigracet 38 BC, FuelCell Store) was non-woven with a microporous layer that was treated with 5\% PTFE. The materials for electron beam deposition were ordered from Plasmaterials. The Au target was $99.99 \%$ pure with 3-6 mm random size pieces, Ag was 99.99\% pure with 3-6 mm random size pieces, and Ti was 99.995\% pure in 0.25 diameter pellets. All water used for experiments was deionized and filtered through a $0.22 \mu \mathrm{m}$ Millipak Express 40, serial number 0826. 
Fabrication of $\mathrm{Au}_{\mathrm{x}} \mathrm{Ag}_{1-\mathrm{x}}$ alloy on carbon paper or silicon:

All electrodes were fabricated with an AMOD dual electron beam deposition system (System 02520, Angstrom Engineering). Carbon paper substrates were lightly dusted under an $\mathrm{N}_{2}$ stream before insertion into the electron beam deposition chamber. Si substrates were cleaned by sonicating sequentially in acetone, isopropanol, and deionized water for two minutes each, stored in deionized water and dried under a stream of $\mathrm{N}_{2}$ prior to use. For the planar gold electrodes on silicon, $2 \mathrm{~nm}$ of Ti was deposited at a rate of $1 \AA / \mathrm{s}$, then $300 \mathrm{~nm}$ of Au was deposited at a rate of $1 \AA / \mathrm{s}$. For the nanoporous gold samples of varying atomic percent gold, $\mathrm{Au}$ and $\mathrm{Ag}$ were co-deposited at varying rates/thicknesses to yield the desired $\mathrm{Au}_{\mathrm{x}} \mathrm{Ag}_{1-\mathrm{x}}$ alloy. For example, a $25 \% \mathrm{Au}$ and $75 \% \mathrm{Ag}$ alloy was fabricated by depositing $75 \mathrm{~nm}$ of $A u$ at a rate of $1 \AA / \mathrm{s}$ and $225 \mathrm{~nm}$ of $A g$ at a rate of $3 \AA / \mathrm{s}$, respectively. Over the course of the deposition the partial pressure of the chamber was maintained between $\sim 10^{-7}$ torr to $\sim 10^{-6}$ torr.

\section{Fabrication of nanoporous gold (np-Au) electrodes by chemical dissolution of Ag:}

$T$ The $\mathrm{Au}_{\mathrm{x}} \mathrm{Ag}_{1-\mathrm{x}}$ alloy coated carbon paper or silicon substrates were placed in a covered glass petri dish of room temperature concentrated nitric acid ( 70 weight/volume) for 15 minutes. A notable color transition from white gold to burnt umber occurs as the silver in the base alloy is removed to yield the desired np-Au morphology. Samples were then removed from the nitric acid and rinsed under a copious flow of water and then soaked in a water bath for an hour. Finally, the substrates were dried under a stream of $\mathrm{N}_{2}$ and subsequently dried in vacuo for 24 hour.

\section{Substrate characterization by Scanning Electron Microscopy (SEM):}

Substrates were imaged in a Nova200 Nanolab Dualbeam FIB/SEM with an acceleration voltage of $10 \mathrm{keV}$ and spot size of 3 . To prepare the a sample for cross sectional imaging, the np-Au coated carbon paper substrate was soaked in liquid $\mathrm{N}_{2}$, cracked in half, and placed on a holder that allowed a $90^{\circ}$ orientation relative to the electron beam. 

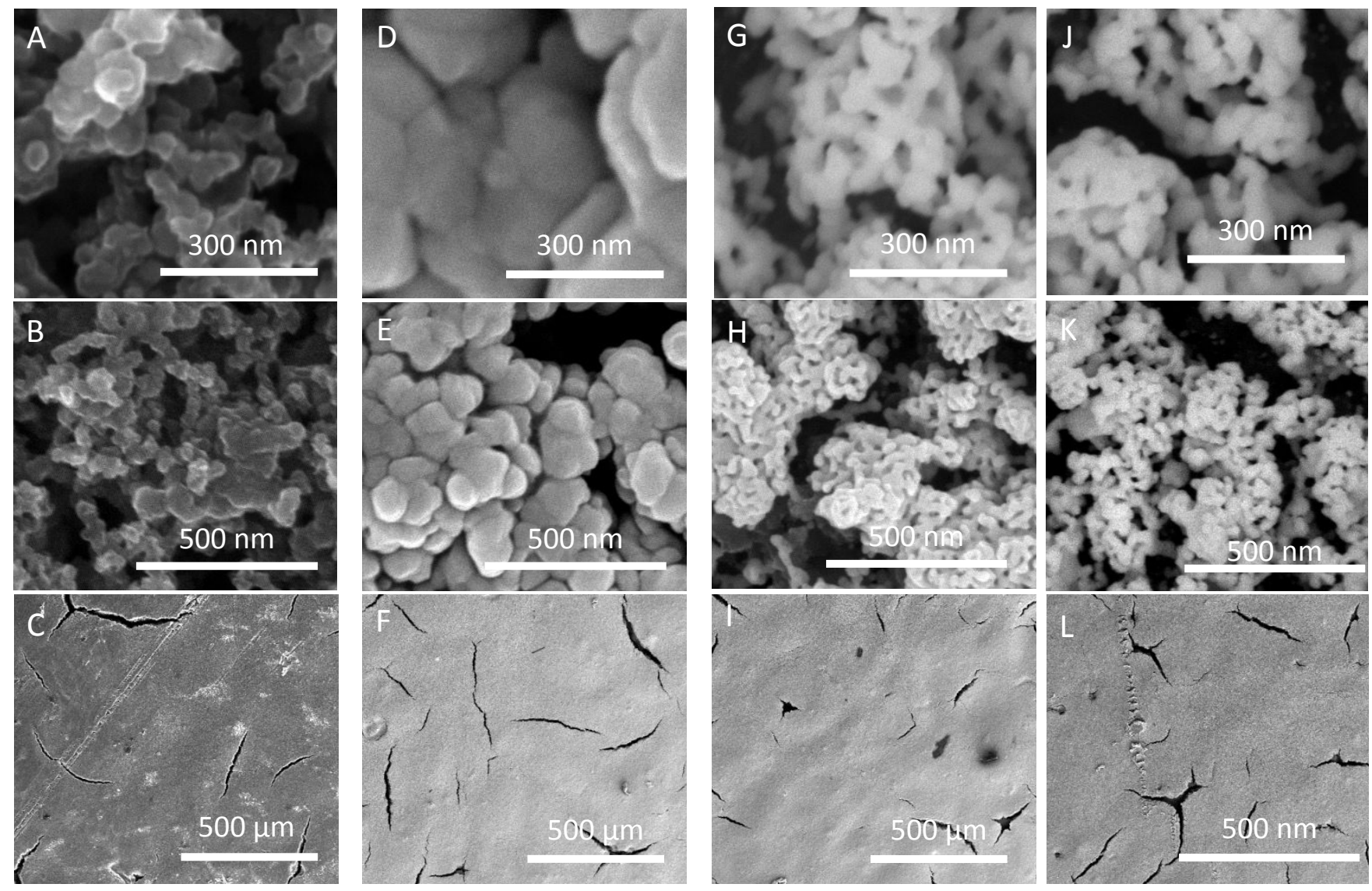

Figure S1: Shows SEM images of the electrode at different phases in the fabrication process. (a)-(c) shows images of the bare carbon paper, Sigracet 38BC. (d)-(f) show images of the gold silver alloy on the carbon paper. (g)-(i) show images of the nanoporous gold morphology from a 35\% Au alloy that forms after the nitric acid etch. (j)-(k) show images of the electrodes after electrolysis. 

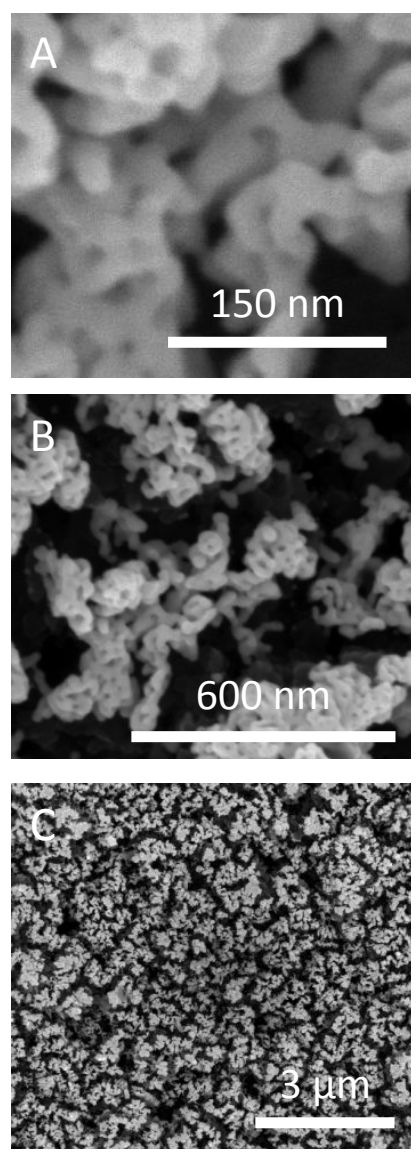
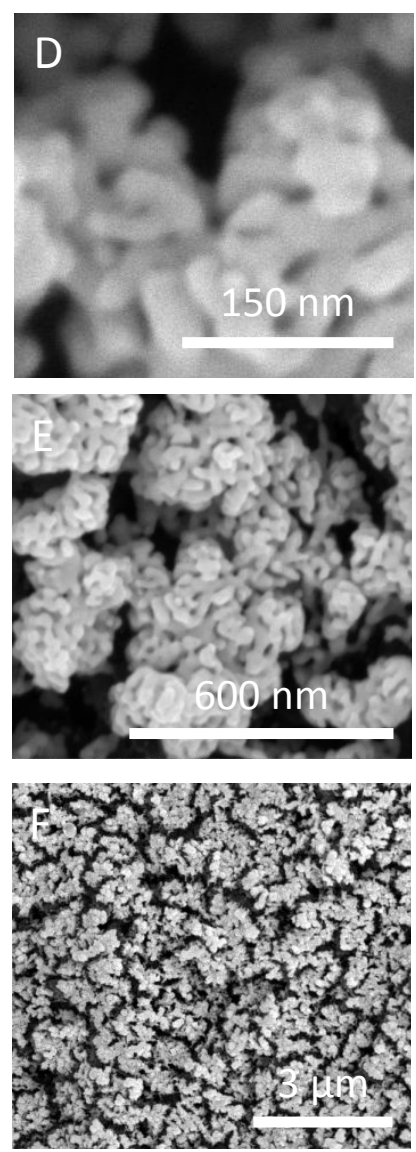
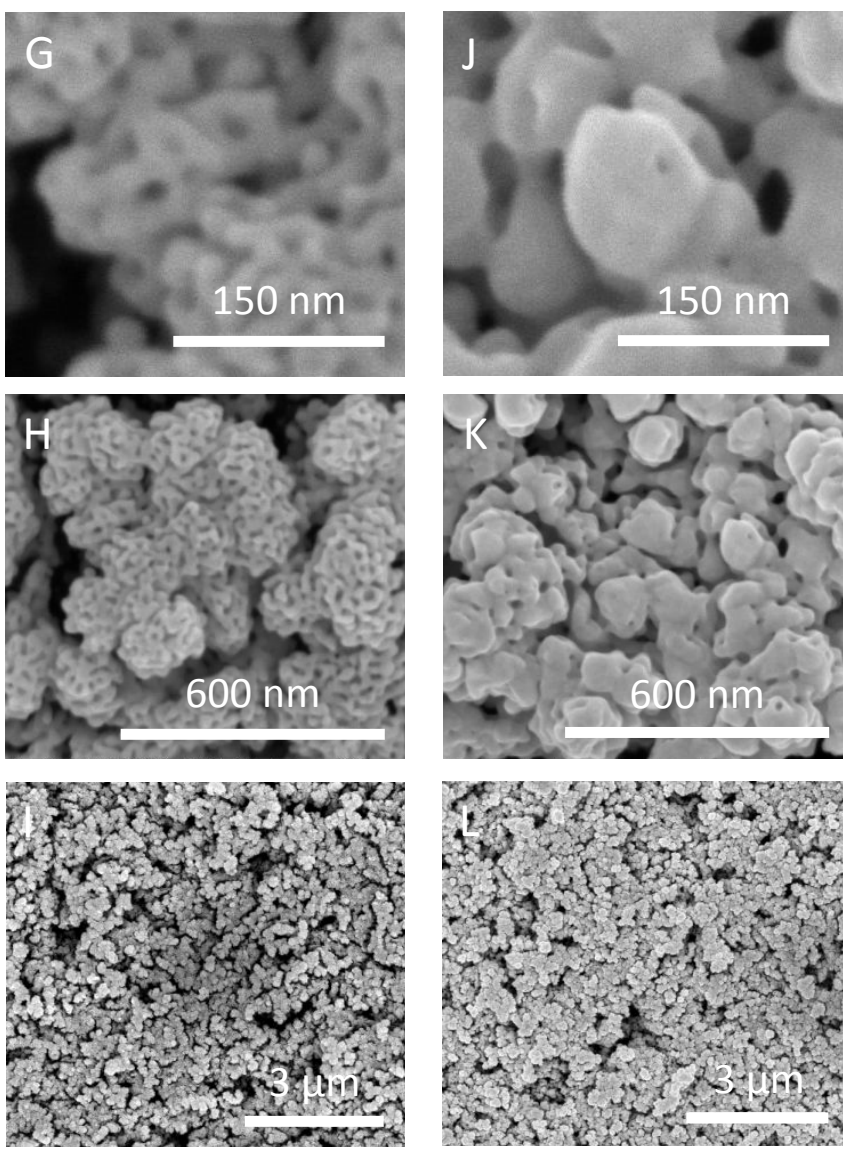

Figure S2: SEM characterization of nanoporous gold (np-Au) electrodes with a varying gold atomic percent $\left(\%_{\mathrm{Au}}\right)$ of $15 \%_{\mathrm{Au}}(\mathrm{a}-\mathrm{c}), 25 \%_{\mathrm{Au}}(\mathrm{d}-\mathrm{f}), 35 \%_{\mathrm{Au}}(\mathrm{g}-\mathrm{i})$, and $45 \%_{\mathrm{Au}}(\mathrm{j}-1)$. 

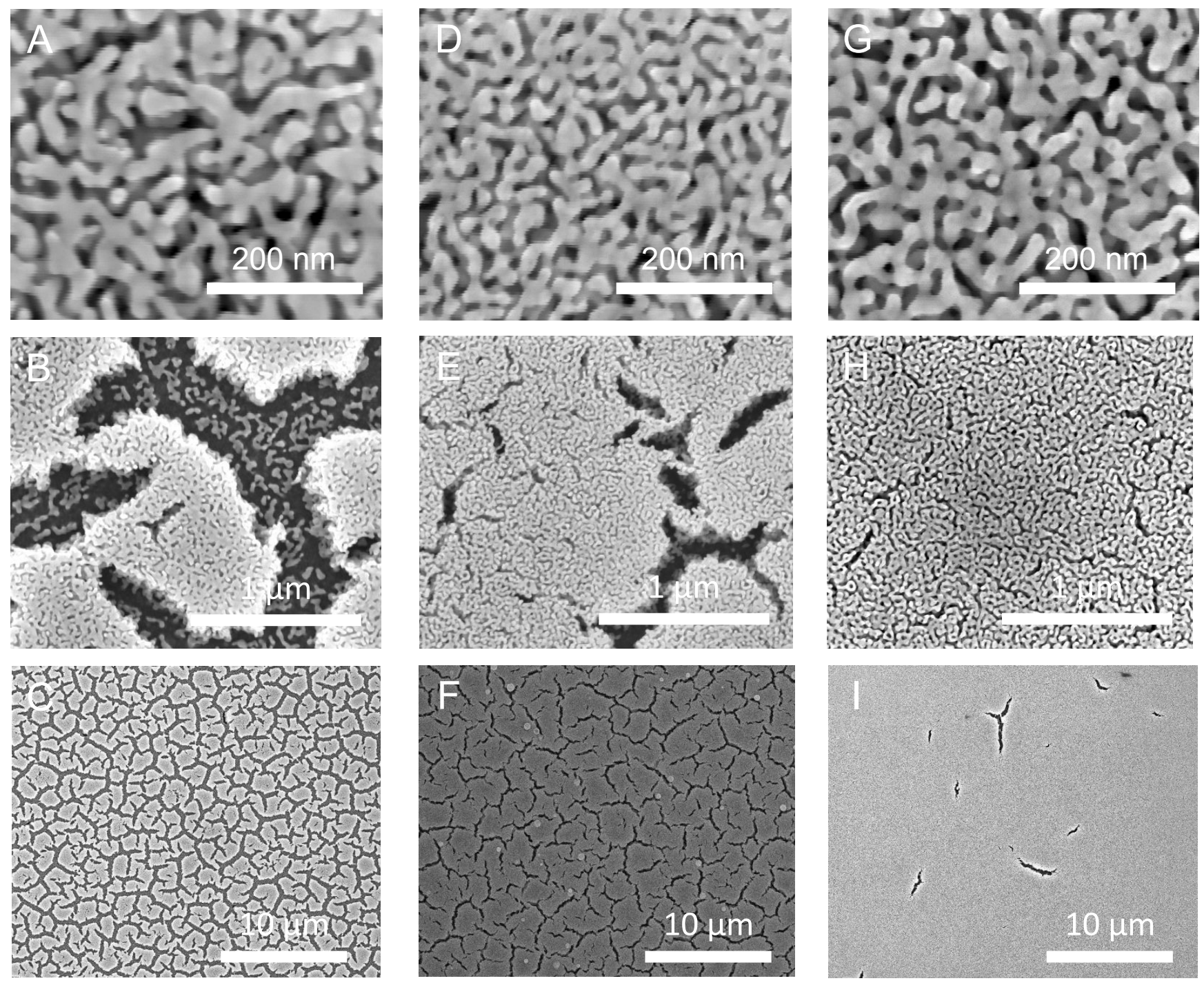

Figure S3: SEM characterization of nanoporous gold (np-Au) electrodes with a varying gold atomic percent $\left(\%_{\mathrm{Au}}\right)$ on silicon. $20 \%_{\mathrm{Au}}(\mathrm{a}-\mathrm{c}), 25 \%_{\mathrm{Au}}(\mathrm{d}-\mathrm{f})$, and $30 \%_{\mathrm{Au}}(\mathrm{g}-\mathrm{i})$. 


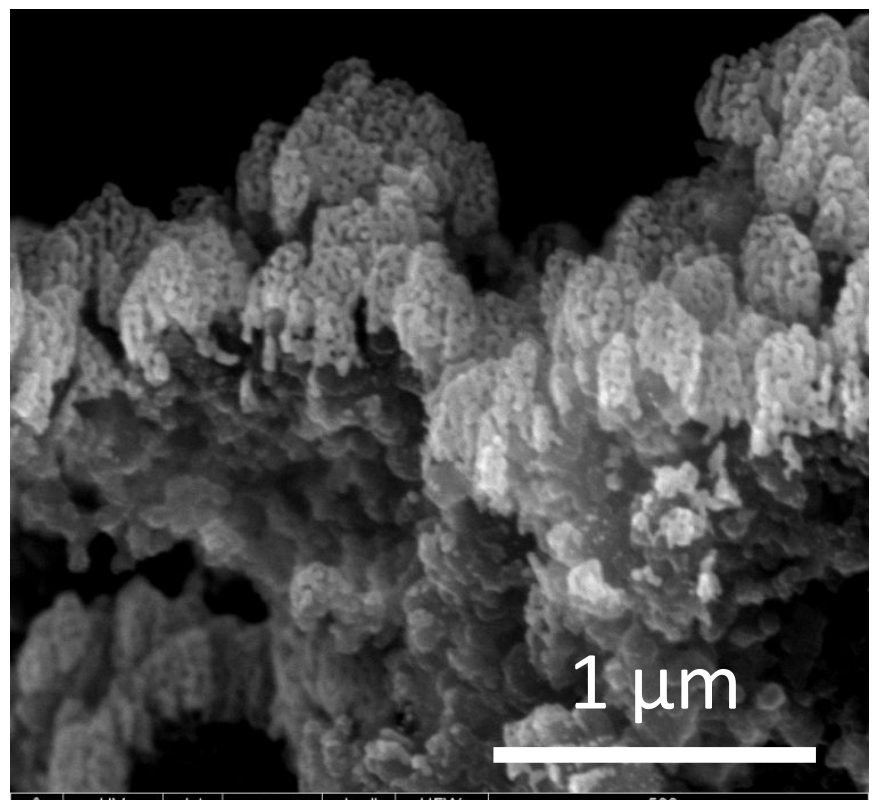

Figure S4: Cross sectional SEM of a $300 \mathrm{~nm}$ thick 35\% gold nanoporous gold electrode on carbon paper. It was observed that while the carbon paper substrate has a high rugosity and is quite uneven, the nanoporous gold had a homogenous pore structure through the entire thickness. 


\section{Electrochemical carbon dioxide reduction $\left(\mathrm{CO}_{2} \mathrm{R}\right)$ experimental set up:}

Electrochemical $\mathrm{CO}_{2} \mathrm{R}$ was performed in a custom flow cell configuration that consisted of a two-compartment cell made of polyether ether ketone (PEEK) and separated with a laser cut Viton rubber gaskets. The tapered catholyte chamber was 3D printed (Creality3D CR-10 3D printer) out of acrylonitrile butadiene styrene (ABS). This chamber design was found to reduce bubble buildup in the catholyte chamber. The carbon paper electrodes were taped with $3 \mathrm{M}$ double sided $\mathrm{Cu}$ tape onto the PEEK back plate with a serpentine channel carved into it. The carbon paper GDE served as the working electrode with an $\mathrm{Ag} / \mathrm{AgCl}$ leakless reference electrode (Innovative Instruments LF-2, 2mm OD) and a platinum mesh counter electrode. The cathode and anode chambers were separated by an anion exchange membrane (AGC, Selemion AMV). A $20 \mathrm{~mL}$ volume of $1 \mathrm{M} \mathrm{KHCO}_{3}$ was separately added to a catholyte and anolyte reservoir and independently recirculated through each respective chamber at flow rate of $15 \mathrm{~mL} / \mathrm{min}$ (two Masterflex 77120-62 pumps). The $1 \mathrm{M} \mathrm{KHCO}_{3}$ electrolyte was bubbled with $\mathrm{CO}_{2}$ (Research grade from Airgas) for 30 minutes minimum prior to use. $\mathrm{CO}_{2}$ was humidified by a glass fritted water bubbler prior to entrance into the electrolysis cell to prevent evaporation of the electrolyte. $\mathrm{CO}_{2}$ was flowed across the back of the carbon paper cathode through the serpentine channel at a flow rate of 50 standard cubic centimeters per minute (SCCM) via an Alicat M-Gas mass flow controller and monitored downstream by an Alicat M-Gas mass flow meter. The cathode outlet gas was sent back to the catholyte reservoir to catch any breakthrough of electrolyte through the GDE. From there the outflowing gas was sent through a $10 \mathrm{~mL}$ water trap to prevent any water from entering the gas chromatograph (SRI-8610) equipped a Haysep D and Molsieve 5A columns and with a thermonal conductivity detector (TCD) and flame ionization detector (FID). Product gas was passed through a methanizer prior to the FID. The electrolyte of the anode and cathode were sampled to measure liquid producs by high performance liquid chromatography (HPLC) however, no liquid products were ever observed.

Electrochemical experiments were performed at room temperature using a potentiostat (Biologic VSP-300). All potentials were converted to the reversible hydrogen electrode (RHE) scale using the following equation: $\mathrm{V}_{\mathrm{RHE}}=\mathrm{V}_{\mathrm{Ag} / \mathrm{AgCl}}+0.197+0.059 *(\mathrm{pH}$ of the medium). Prior to each experiment, potentiostatic impedance spectroscopy (PEIS) was carried out to determine the solution resistance of the cell (typically between 5-10 $\Omega$ ). The applied potential was compensated $85 \%$ by the potentiostat and remainder determined by the resistance form PEIS. When an operating cell potential was applied, the current was stabilized for 200 seconds minimum before any GC analysis was carried out. The current density was averaged for the 150 seconds prior to the GC injection. After an electrochemical run, the cell was disassembled, sonicated in 15\% nitric acid for 10 minutes, rinsed with water and sonicated in water for 10 minutes prior to reuse at least 2 times. The cell was stored in a $15 \%$ nitric acid solution at the end of each day. Each electrode was disposed of after a single run. Carbon paper controls in $1 \mathrm{M}$ $\mathrm{KHCO}_{3}$ were carried out. At $-1.48 \mathrm{~V}_{\mathrm{Ag} / \mathrm{AgCl}}$ we observed a $0.63 \mathrm{~mA} / \mathrm{cm}^{2}$ current density that went only towards HER. At $-1.68 \mathrm{~V}_{\mathrm{Ag} / \mathrm{AgCl}}$ we observed a $1.2 \mathrm{~mA} / \mathrm{cm}^{2}$ current density that consisted entirely of HER. No $\mathrm{CO}_{2} \mathrm{R}$ products were detected by $\mathrm{GC}$ and thus we conclude that all $\mathrm{CO}$ observed in our experiments arises from genuine $\mathrm{CO}_{2} \mathrm{R}$ carried out by our np-Au catalysts. 


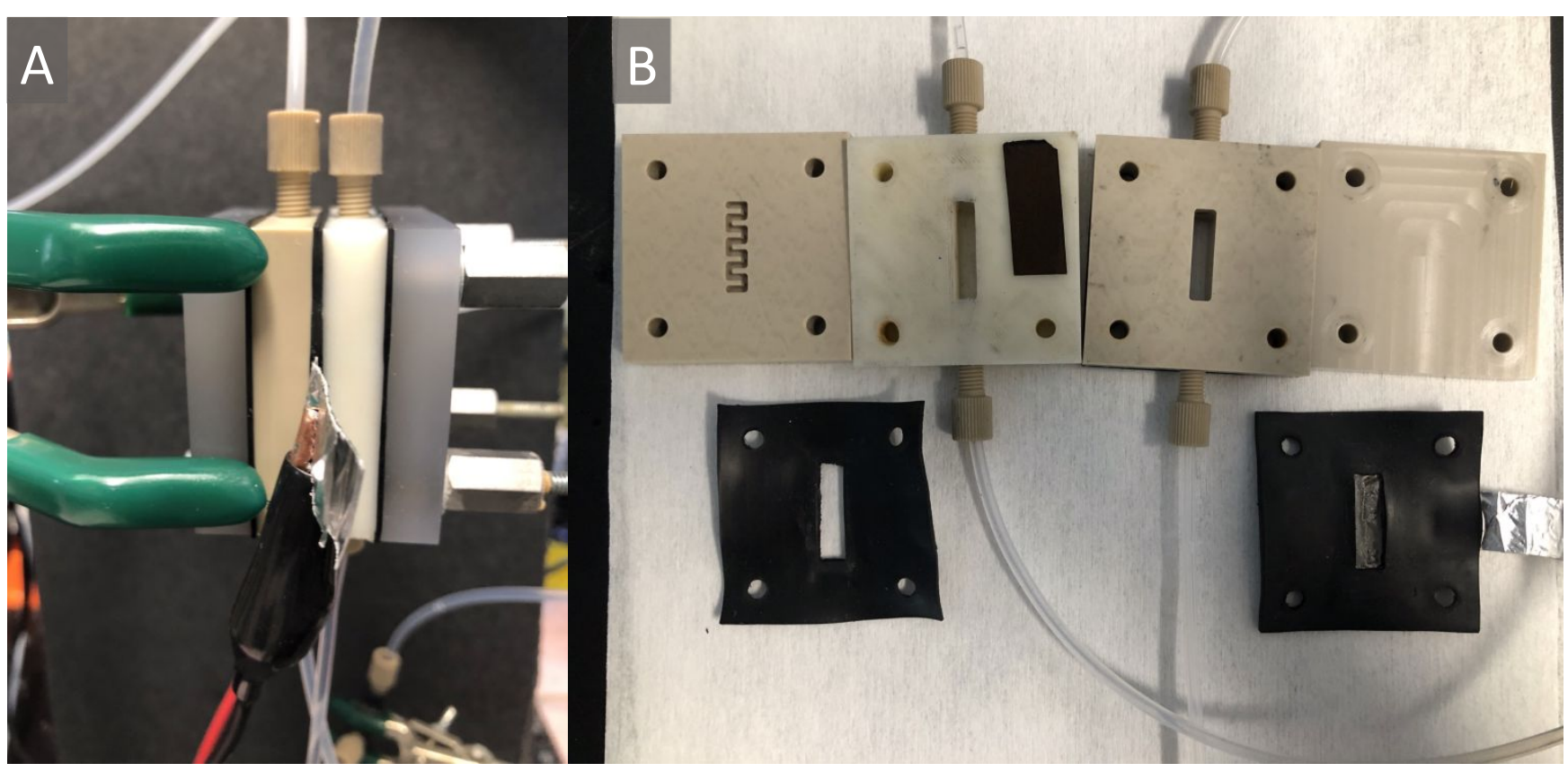

Figure S5: Photographs of electrochemical cell and experimental set up. (A) shows the cell set up for operation with leads attached. (B) shows each individual component of the disassembled cell. From left to right there is the serpentine channel, 3D printed catholyte chamber (with np-Au electrode on top), anode chamber and anode back plate. Below are the laser cut Viton gaskets with the gaskets. The left gasket has the Pt mesh counter taped behind it with an Al lead attached. 


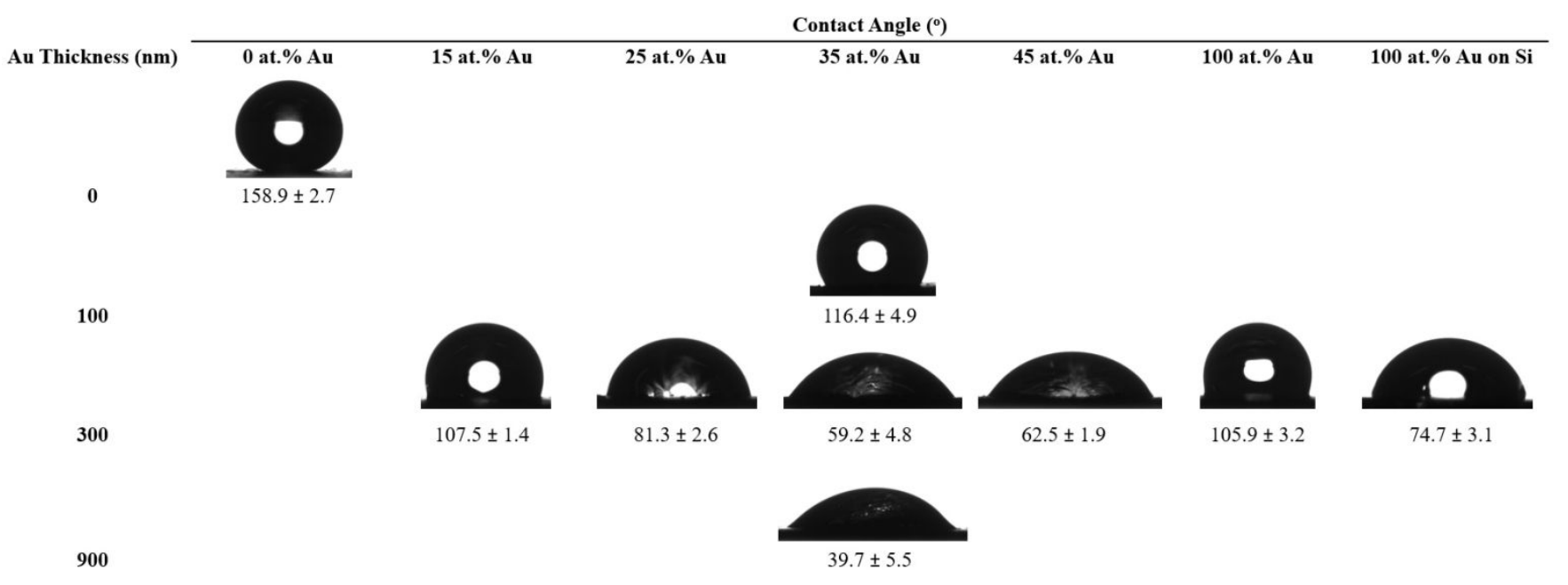

Figure S6: Contact angle measurements of nanoporous gold electrodes with varying atomic gold percentages. 0 atomic percent $\mathrm{Au}$ indicates that there is no catalyst layer and 100 atomic percent $\mathrm{Au}$ indicates that there was a solid gold film deposited. All samples were deposited on Sigracet 38BC unless otherwise noted. 


\section{Cu underpotential deposition (Cu UPD) measurements:}

$\mathrm{Cu}$ UPD measurements were utilized to determine the surface area of an electrode in contact with the electrolyte. $\mathrm{A} \mathrm{CuSO}_{4}$ solution $(0.1 \mathrm{M})$ in $0.5 \mathrm{M} \mathrm{H}_{2} \mathrm{SO}_{4}$ was used as the deposition bath for all $\mathrm{Cu}$ UPD experiments. The solution was sparged with $\mathrm{N}_{2}$ (research grade form Airgas) for 30 minutes to remove dissolved $\mathrm{O}_{2}$ prior to any experiment. The working electrode was a planar $\mathrm{Au}$ or np-Au film of various thickness on carbon paper or a flat silicon wafer with a $\mathrm{Pt}$ mesh counter electrode and an $\mathrm{Ag} / \mathrm{Ag} / \mathrm{Cl}$ reference electrode. Flooded measurements were carried out in a single chamber compression cell and in situ GDE measurements were carried out in a single chamber compression cell in which $\mathrm{N}_{2}$ was flowed at various flow rates through a serpentine channel behind the electrode. CVs from $450 \mathrm{mV}$ to 50 $\mathrm{mV}$ vs $\mathrm{Ag} / \mathrm{AgCl}$ at a scan rate of $5 \mathrm{mV} / \mathrm{sec}$ were acquired until the traces converged. A total of three electrodes were measured at each thickness and flow rate. The anodic stripping peak was integrated to determine charge passed. The electrochemical surface area enhancement was obtained by taking the average surface area of the each electrode relative to a planar Au film deposited onto a flat Si wafer.

\section{Secondary Ion Mass Spectroscopy (SIMS) sample preparation and measurements:}

The depth profiles of the samples were collected with a Cameca ims 7f-GEO secondary ion mass spectrometer (SIMS) at the Caltech Microanalysis Center. A rastering (100 um x $100 \mathrm{um}$ ) O2+ primary beam $(+13 \mathrm{keV}, 15 \mathrm{nA})$ was used to sputter the sample surface. Positive secondary ions of $7 \mathrm{keV}$ were extracted and collected in peak-jumping mode with either an electron multiplier (EM, for $12 \mathrm{C}+; 65 \mathrm{Cu}+$; $107 \mathrm{Ag}+$; and $197 \mathrm{Au}+)$ or a Faraday Cup $(\mathrm{FC}$, for $65 \mathrm{Cu}+)$. A field aperture was used to avoid edge effect from beam sputtering and to limit collected secondary ions only from the center area of 35 um in diameter. In each data collection cycle, the collection time was $1 \mathrm{sec}$ for each mass with appropriate magnet settling time in between. Because there were no significant interferences for the masses being measured, the mass resolving power (MRP) of the mass spectrometer was set at 1200 . The np-Au electrode analyzed by SIMS were prepared by putting each electrode into the Cu UPD deposition bath in the desired cell configuration and electroplating $\mathrm{Cu}$ onto the electrodes by holding a constant potential of $-0.08 \mathrm{~V}$ vs $\mathrm{Ag} / \mathrm{AgCl}$ for 45 seconds for each distinct electrode. The residual Ag in the fully etched np$\mathrm{Au}$ electrodes was determined by SIMS as well. The silver content was calibrated with a $15 \%$ Au and $35 \%{ }_{\mathrm{Au}}$ white gold alloy and the residual $\mathrm{Ag}$ in an etched $35 \% \mathrm{Au}$ np-Au electrode was determined to be $1.3 \%$.
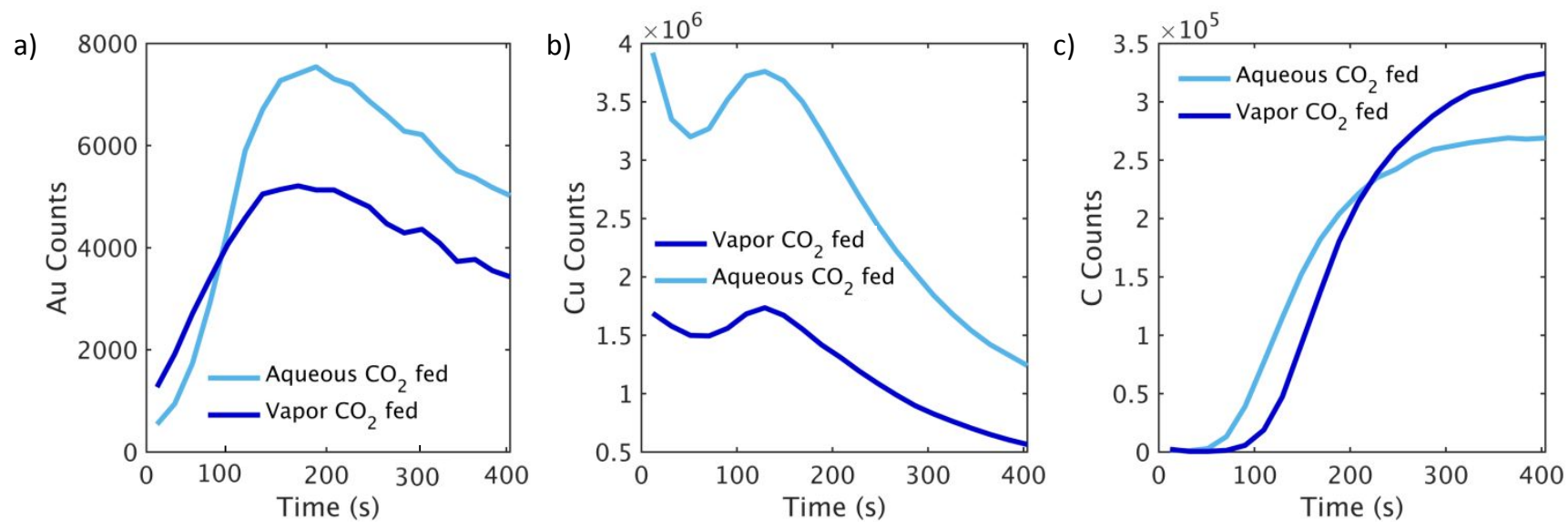
Figure S7: Secondary ion mass spectroscopy raw data for aqueous $\mathrm{CO}_{2}$ fed system and vapor $\mathrm{CO}_{2}$ fed system. (a) shows the gold counts, (b) the copper counts, and (c) the carbon counts. In conjunction with figure 5 of the main text, we hypothesize the $\mathrm{Cu}$ :Au counts decrease as a function of depth in both samples as consequence of the rough and bumpy nature of the underlying carbon paper substrate. We hypothesize the $\mathrm{Cu}$ :Au ratio is enhanced at the surface of the catalyst layer because at this location (and time) we are only sputter profiling through pure $\mathrm{Cu}$ and $\mathrm{Au}$. As we penetrate deeper into the catalyst layer, we are now also sputter depth profiling through some of the underlying carbon. The copper is not going to plate onto the unflooded carbon, so it appears that there is less copper deeper in the substrate. This is supported by figure S7 C, where after 100 seconds the carbon counts steadily increase.
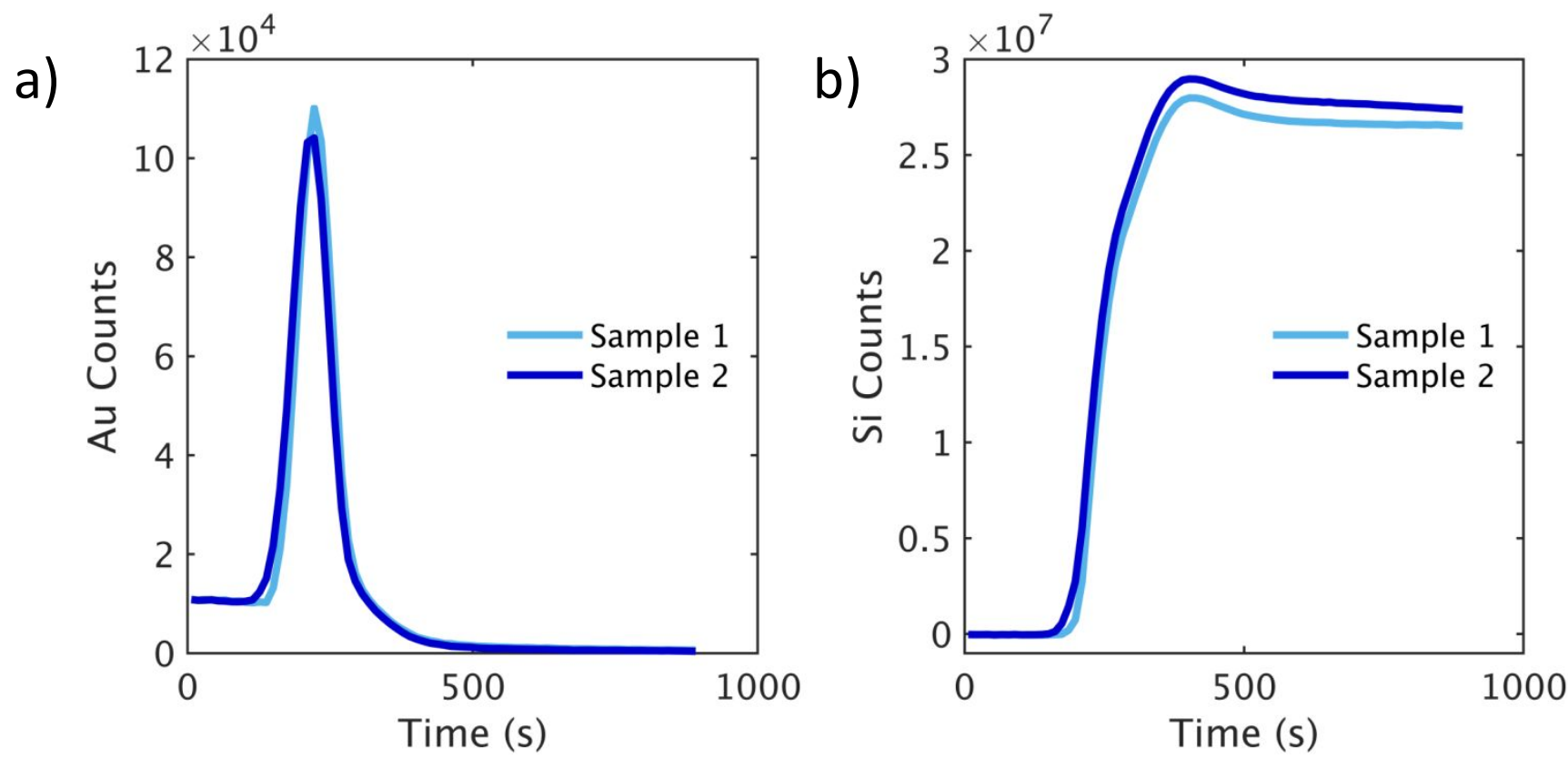

Figure S8: Secondary ion mass spectroscopy raw data for $300 \mathrm{~nm}$ planar Au on a silicon wafer (a) shows the Au counts, and (b) shows the Si counts. 


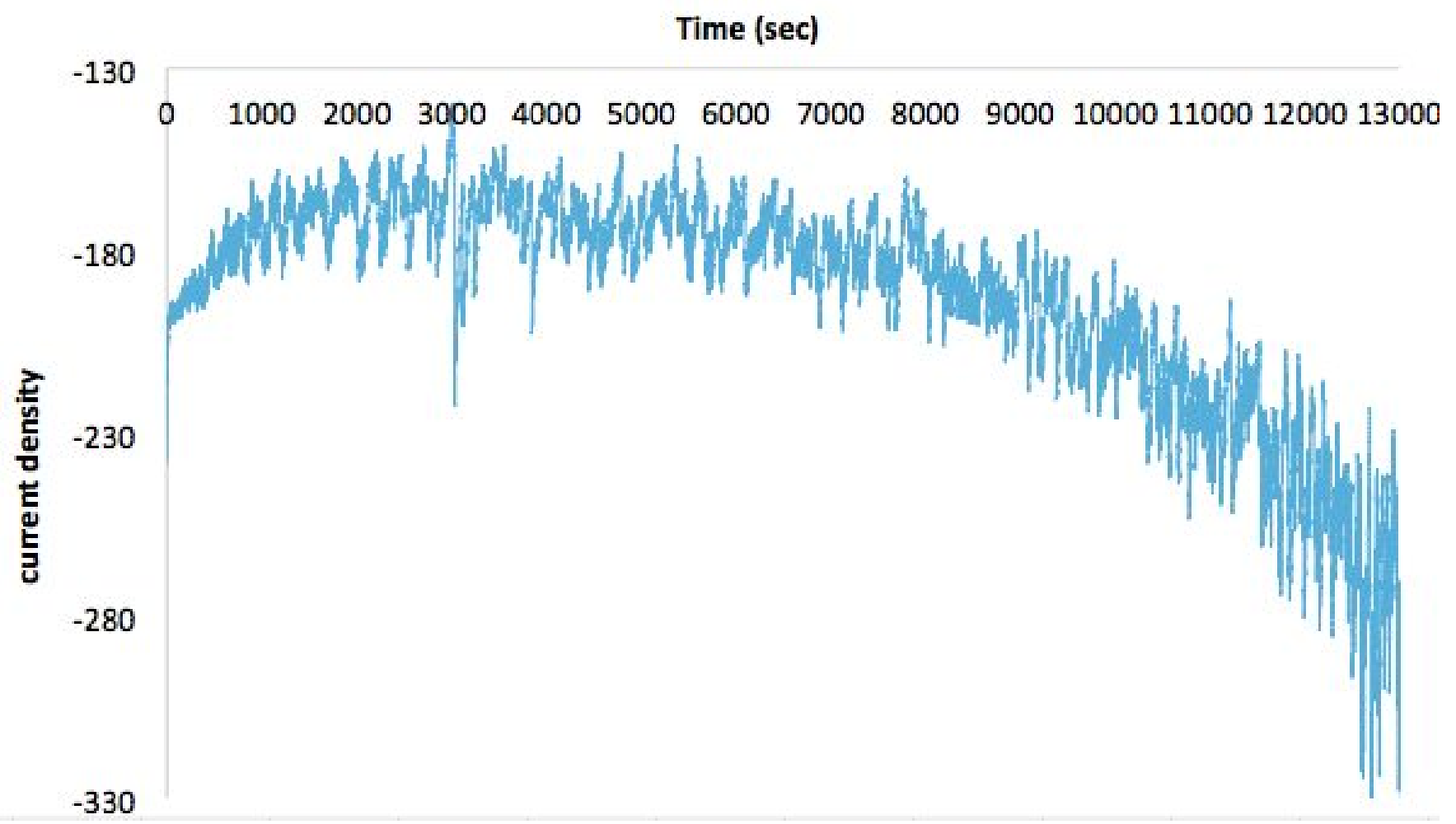

Figure S9: Long term chronoamperometry of a $25 \%$ Au electrode run at $50 \mathrm{SCCM}$ of $\mathrm{CO}_{2}$ in a $1 \mathrm{M}$ $\mathrm{KHCO}_{3}$ electrolyte. The $\mathrm{x}$ axis is time in seconds and the $\mathrm{y}$ axis is current density in $\mathrm{mA} / \mathrm{cm}^{2}$. Injections were taken at every 15 minutes starting at 5 minutes in. The first three injections have an averaged $\mathrm{FE}_{\mathrm{CO}}$ of $76 \%$. The electrode is quite stable for the first hour of operation (the large spikes in current density are bubbles forming and coming off the electrode surface). After the first $\sim 50$ minutes of operation the carbon paper substrate begins to flood and HER increases at the expense of HER. The fourth injection has $\mathrm{FE}_{\mathrm{CO}}$ of $71 \%$. At 2 hours of operation the electrolyte was observed to break through the carbon paper substrate and into the serpentine channel gas line. Carbon paper is well known to degrade after an hour and this is not unexpected. HER continues to increase to over $50 \%$ of the current density throughout the duration of the experiment. We note that all the experiments presented in the main text of this paper were taken during the first 45 minutes of operation of any electrode. 